\title{
In vitro Quality of Platelets with Low Plasma Carryover Treated with Ultraviolet C Light for Pathogen Inactivation
}

\author{
Lacey Johnson $^{a}$ Ryan Hyland ${ }^{a}$ \\ Shereen Tan ${ }^{a}$ Frank Tolksdorf ${ }^{b}$ \\ Axel Seltsam $^{d}$ Denese Marks ${ }^{a}$

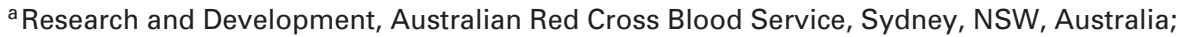 \\ b MacoPharma International GmbH, Langen, Germany; \\ cMacoPharma, Tourcoing, France; \\ dGerman Red Cross Blood Service NSTOB, Springe, Germany
}

Chryslain Sumian ${ }^{\mathrm{c}}$

\section{Keywords}

UVC - Pathogen inactivation - Platelet concentrates .

Plasma carryover

\section{Summary}

Background: The THERAFLEX UV-Platelets system uses shortwave ultraviolet C light (UVC, $254 \mathrm{~nm}$ ) to inactivate pathogens in platelet components. Plasma carryover influences pathogen inactivation and platelet quality following treatment. The plasma carryover in the standard platelets produced by our institution are below the intended specification ( $<30 \%)$. Methods: A pool and split study was carried out comparing untreated and UVC-treated platelets with $<30 \%$ plasma carryover ( $n=10$ pairs). This data was compared to components that met specifications ( $>30 \%$ plasma). The platelets were tested over storage for in vitro quality. Results: Platelet metabolism was accelerated following UVC treatment, as demonstrated by increased glucose consumption and lactate production. UVC treatment caused increased externalization of phosphatidylserine on platelets and microparticles, activation of the GPIIb/IIla receptor (PAC-1 binding), and reduced hypotonic shock response. Platelet function, as measured with thrombelastogram, was not affected by UVC treatment. Components with $<30 \%$ plasma were similar to those meeting specification with the exception of enhanced glycolytic metabolism. Conclusion: This in vitro analysis demonstrates that treatment of platelets with $<30 \%$ plasma carryover with the THERAFLEX UV-Platelets system affects some aspects of platelet metabolism and activation, although in vitro platelet function was not negatively impacted. This study also provides evidence that the treatment specifications of plasma carryover could be extended to below $30 \%$.

(c) 2015 S. Karger GmbH, Freiburg

\section{Introduction}

Pathogen inactivation (PI) methods attempt to prevent the replication of pathogens in blood components, thereby reducing the risk of transfusion-transmitted infection (reviewed in [1]). The INTERCEPT and Mirasol systems use ultraviolet (UV) light in conjunction with a photosensitizing agent, whereas the THERAFLEX UV-Platelets system (referred to herein as UVC treatment) does not utilize a photosensitizer, but relies on shortwave UVC light $(254 \mathrm{~nm})$. It is well established that UVC light alone can be microbiocidal and virucidal, inducing DNA damage through the dimerization of adjacent pyrimidine nucleotides [2-4]. However, turbid solutions such as platelet concentrates have low permeability to UVC light [4]. As such, UVC treatment requires the preparation of platelets in platelet-additive solution (PAS) as well as strong agitation to form shallow regions within the platelet component to facilitate sufficient light penetration.

The THERAFLEX UV-Platelets system has been CE-marked, and several investigations have been conducted to determine the effect of UVC treatment on in vitro platelet quality [4-8]. Treatment with the UVC system appears to accelerate the metabolic rate of platelets, which is similar to the effects of other PI methods [911]. However, in vivo investigations using the UVC system are showing positive results $[5,12]$.

To balance PI and minimize platelet damage, the optimal light dose must be achieved. The dose of light effectively applied to a

\section{KARGER \\ Fax +497614520714

\section{(c) 2016 S. Karger GmbH, Freiburg}

1660-3796/16/0433-0190\$39.50/0 
Table 1. Specifications of platelet concentrates*

\begin{tabular}{|c|c|c|c|c|c|c|c|}
\hline \multirow[t]{2}{*}{ Parameter } & \multirow[t]{2}{*}{ Specifications } & \multicolumn{3}{|c|}{$<30 \%$ plasma carryover } & \multicolumn{3}{|c|}{$>30 \%$ plasma carryover } \\
\hline & & control & UVC-treated & p value ${ }^{\ddagger}$ & control & UVC-treated & p value $e^{\ddagger}$ \\
\hline Platelets $\times 10^{9} / \mathrm{ml}$ & $0.8-1.4$ & $0.86 \pm 0.06$ & $0.86 \pm 0.07$ & 0.7655 & $0.83 \pm 0.08$ & $0.84 \pm 0.09$ & 0.3576 \\
\hline Volume, ml & $325-375$ & $353.1 \pm 6.1$ & $353.6 \pm 6.0$ & 0.6599 & $369.8 \pm 4.7$ & $369.8 \pm 6.3$ & 0.8433 \\
\hline Plasma carryover, \% & $30-40$ & $27.4 \pm 1.7$ & $27.7 \pm 1.8$ & 0.6459 & $31.8 \pm 1.0^{\dagger}$ & $32.2 \pm 1.3^{\dagger}$ & 0.2327 \\
\hline
\end{tabular}

platelet concentrate is influenced by the platelet concentration and plasma carryover, and as such, the platelet component must meet certain specifications to be suitable for treatment (table 1). The pooled platelet components routinely produced in Australia do not meet all of these specifications. In particular, the plasma carryover is below the lower limit of the specifications for UVC treatment (30-40\%). As such, the aim of this study was to evaluate the in vitro quality of the standard pooled platelet units prepared in Australia following treatment with the UVC system. Further, these data were compared to components which meet the current specifications for UVC treatment. This data is required to provide confidence that these products would be suitable for use in a clinical setting.

\section{Material and Methods}

\section{Platelet Production and UVC Treatment}

This study had approval from the Australian Red Cross Blood Service Human Research Ethics Committee. Buffy coats were prepared from whole blood donations collected in citrate-phosphate-dextrose (CPD) solution [13]. Platelet components were prepared by pooling four $\mathrm{ABO} / \mathrm{RhD}$ matched buffycoats with $300 \mathrm{ml}$ additive solution (SSP+; MacoPharma, Mouvaux, France) within $24 \mathrm{~h}$ of collection. The platelets were then separated by slow centrifugation $(500 \times g ; 6 \mathrm{~min})$ followed by in-line leukofiltration (Imuguard III-S PL filter; TerumoBCT, Somerset, NJ, USA).

On day 1 post-collection, platelet components were prepared using a pool and split design $(\mathrm{n}=10)$. Two platelet components were pooled and split, and one unit was UVC-treated whilst the other remained untreated as a control. All platelets were sterile docked onto an illumination set and transferred into a $19 \times$ $38 \mathrm{~cm}$ illumination bag composed of UVC-permeable ethyl vinyl acetate (EVA). The untreated units were transferred directly into the associated platelet storage bag, which was composed of n-butyryl tri n-hexyl citrate (BTHC) plasticized PVC [14]. The UVC units were treated with the THERAFLEX UVPlatelets system, according to the manufacturer's instructions (MacoPharma). Briefly, the component was fixed to the edges of a quartz plate, agitated at 110 $\mathrm{rpm}$ and illuminated with a dose of $0.2 \mathrm{~J} / \mathrm{cm}^{2} \mathrm{UVC}$ light $(254 \mathrm{~nm}$ ) from the top and bottom. Following treatment, the components were transferred to the associated platelet storage bag and stored at $20-24^{\circ} \mathrm{C}$ with agitation (Helmer Inc., Noblesville, IN, USA)

Platelet samples $(10 \mathrm{ml})$ were removed on day 1 (immediately after treatment), 2, 5, and 7 by sterile docking on a sample pouch.

To compare the low plasma carryover group with components which meet the specifications ( $>30 \%$ plasma) for UVC treatment, an additional study group was prepared $(\mathrm{n}=10)$. To achieve $>30 \%$ plasma carryover, two platelet components were pooled and supplemented with $45 \mathrm{ml}$ of ABO-matched plasma (freshly thawed plasma) prior to splitting. These pairs $(>30 \%)$ were treated and tested in the same way as the standard units.

\section{Component Specifications and Quality}

The platelet count and MPV were measured using a hematology analyzer (CellDyn Emerald; Abbott Laboratories, Abbott Park, IL, USA). Plasma carryover was measured from the supernatant on day 1 using a bicinchoninic acid (BCA) protein assay kit (Pierce Biotechnology, Rockford, IL, USA). The total protein concentration was calculated from a bovine serum albumin (BSA) standard curve, and plasma carryover in the platelet concentrate was calculated as a percentage of the total protein present in $100 \%$ plasma. Supernatants were prepared by double centrifugation at $1,600 \times g$ for $20 \mathrm{~min}$ and then $12,000 \times g$ for $5 \mathrm{~min}$ at room temperature, and stored at $-80^{\circ} \mathrm{C}$ until tested in the assays as described below.

\section{Assessment of Platelet Quality and Function}

The $\mathrm{pH}, \mathrm{pO}_{2}, \mathrm{pCO}_{2}$, and lactate content of platelet components were measured immediately after sampling using an i-STAT blood gas analyzer and CG4+ cartridges (Abbott Diagnostics, Sydney, Australia). These parameters were measured at $37^{\circ} \mathrm{C}$, and bicarbonate was calculated by the i-STAT analyzer. Extracellular glucose was measured from the platelet supernatant using a colorimetric assay measuring the enzymatic conversion of glucose to hexokinase/ glucose-6-phosphate dehydrogenase (Fisher Diagnostics, Middletown, VA, USA). Extracellular lactate dehydrogenase (LDH) was measured from supernatants using an in vitro toxicology assay (Sigma Aldrich, St. Louis, MO, USA), against a standard curve of L-lactic dehydrogenase. Adenosine triphosphate (ATP) concentration was measured using an ATP bioluminescence kit (Roche, Mannheim, Germany), as previously described [9]. Samples for glucose, LDH, and ATP were tested in triplicate against a standard curve.

Changes in platelet mitochondrial transmembrane potential $(\Delta \psi)$ were determined using the lipophilic cationic fluorochrome JC-1 (Biotium Inc., Hayward, CA, USA). The percentage of platelets displaying a polarized mitochondrial membrane (red fluorescence) was measured by flow cytometry, as previously described [9].

The expression of platelet glycoproteins was measured by flow cytometry as previously described [15]. PAC-1 binding was measured at basal levels, where platelets $\left(3 \times 10^{6}\right)$ were stained with $20 \mu \mathrm{l}$ PAC-1-FITC (BD Biosciences, Franklin Lakes, NJ, USA) for $20 \mathrm{~min}$ at room temperature in the dark, and the median fluorescence intensity (MFI) was assessed by flow cytometry. For p-selectin, platelets $\left(3 \times 10^{6}\right)$ were stained with CD62P-PE (BD Biosciences) for $20 \mathrm{~min}$ at room temperature, and percentage of positive cells was assessed compared to an isotype control. For phosphatidylserine exposure, platelets $\left(1 \times 10^{6}\right)$ were stained with $5 \mu$ annexin-V-FITC binding in calcium-containing buffer (BioLegend, San Diego, CA, USA) for $15 \mathrm{~min}$ in the dark, and the percentage of positive cells was reported.

The absolute number of platelet microparticles was determined by flow cytometry using TruCount tubes (BD Biosciences), as previously described [13]. Platelet microparticles were stained with CD61-APC and annexin-V-FITC and defined as events $\leq 1.0 \mu \mathrm{m}$. The procoagulant activity of platelet microparticles was assessed using the STA-Procoag-PPL kit (Diagnostica Stago Ltd, Asnieres, France), as previously described [13].

Cytokine release was measured from the supernatants of platelet concentrates. Commercially available ELISA kits for CD40L, RANTES, soluble CD62P, PDGF-AB, PF4 and NAP2 (as a measure of $\beta$-thromboglobulin) were used ac- 

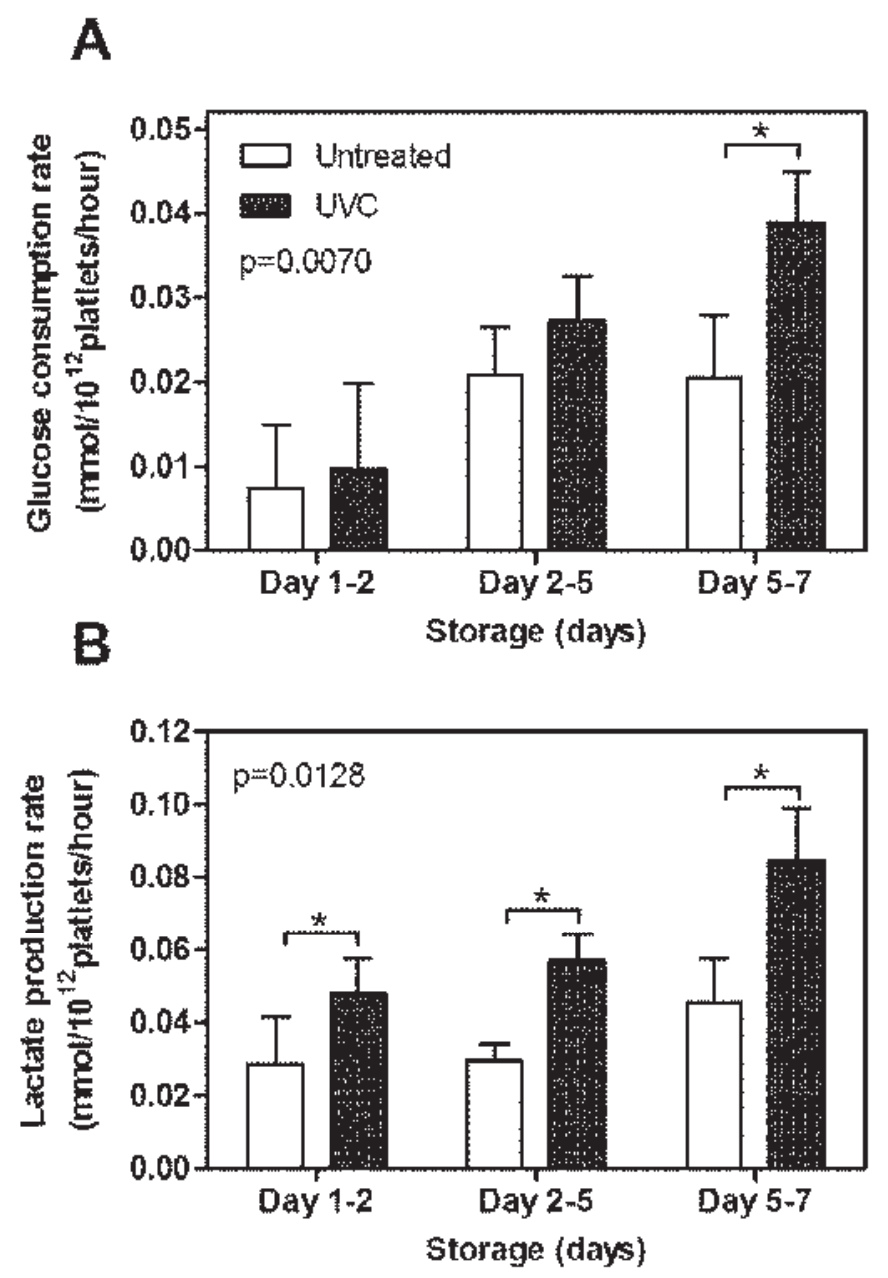

C

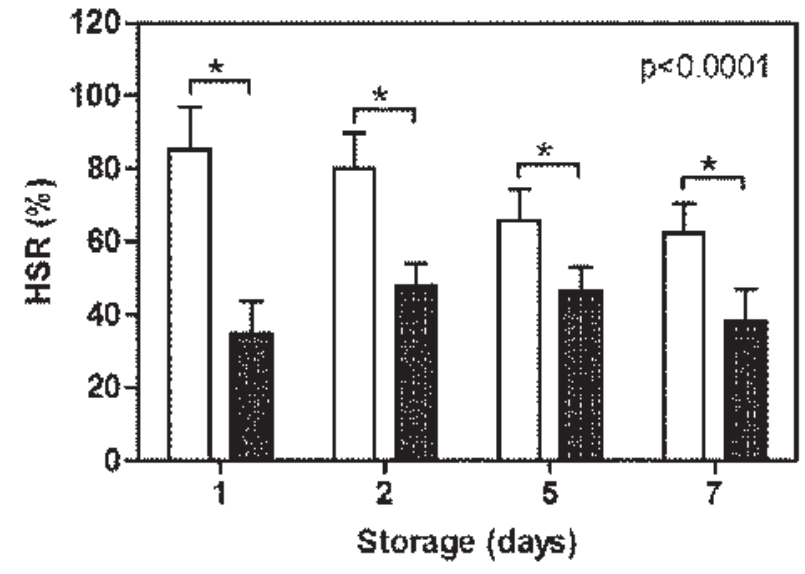

Fig. 1. UVC treatment alters platelet metabolic parameters. Platelet concentrates with $<30 \%$ plasma carryover were treated and stored for 7 days. The concentration of $\mathbf{A}$ glucose and $\mathbf{B}$ lactate were measured and consumption and production rates were calculated using the platelet count and are given as $\mathrm{mmol} / 10^{12}$ platelets/h during the indicated time periods. C HSR was measured using phosphate buffer solution and water. The data represent mean $\pm \mathrm{SD}$ (error bars). The $\mathrm{p}$ value indicates the interaction between storage and treatment as determined by two-way repeated measures ANOVA; ${ }^{*}$ Indicates $\mathrm{p}<0.05$ between untreated and UVC-treated platelets at the same time point.

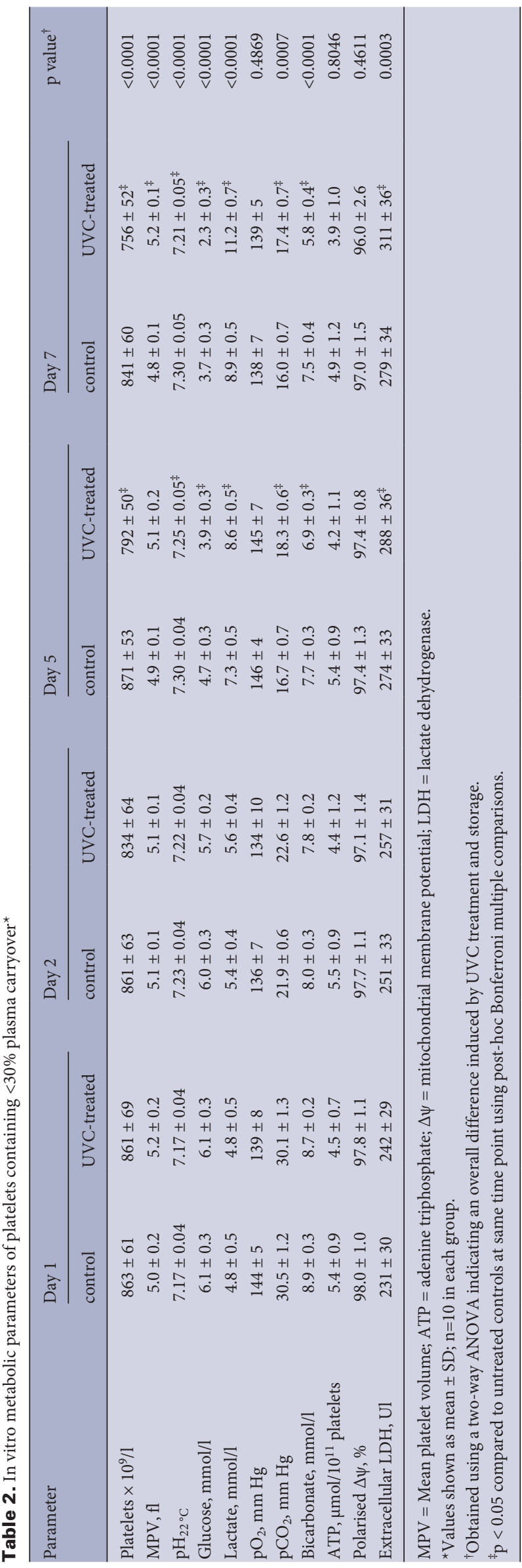

Johnson/Hyland/Tan/Tolksdorf/Sumian/Seltsam/ Marks 
A

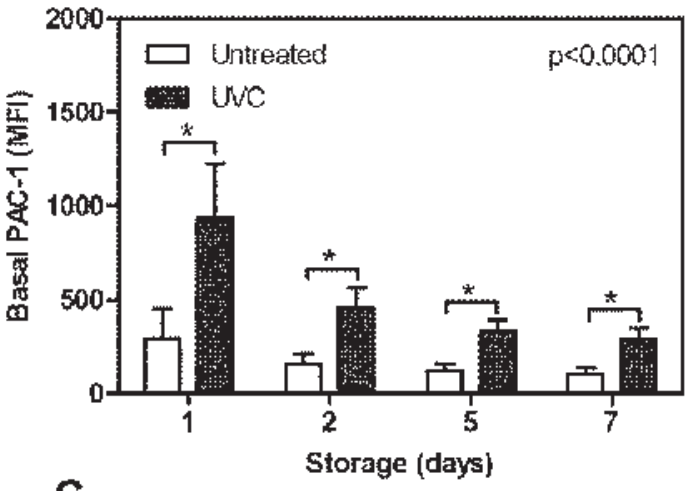

C

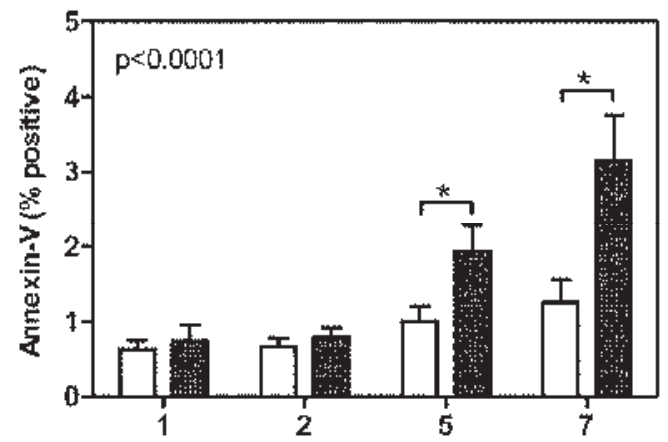

E

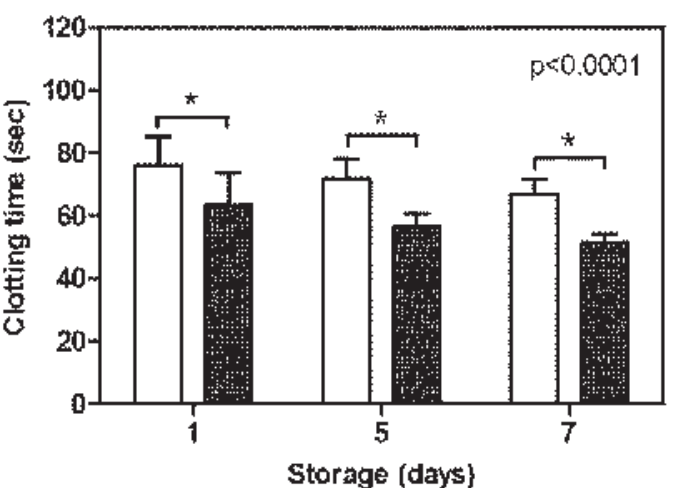

Storage (days)
B

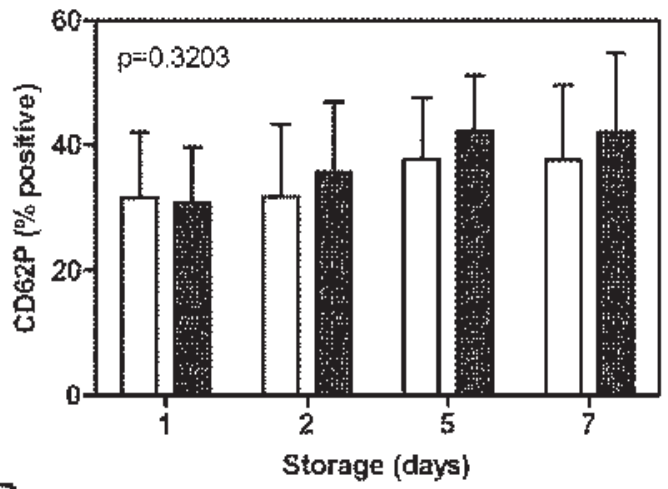

D

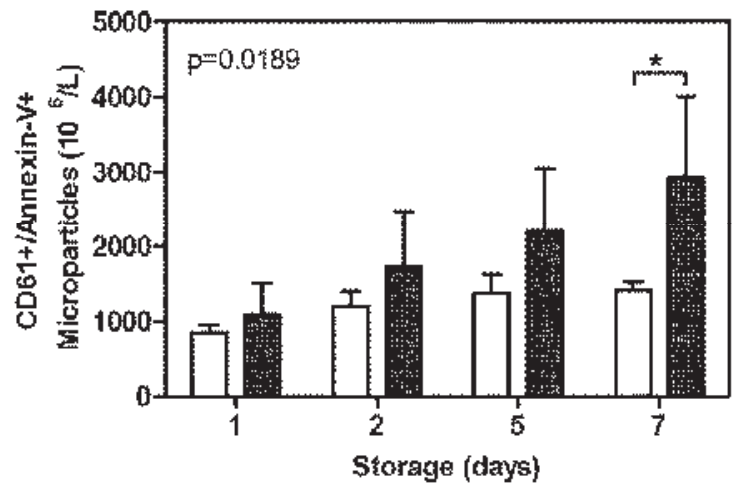

assay using platelet

supernatant. The data represent mean $\pm \mathrm{SD}$ (error bars). The $\mathrm{p}$ value indicates the interaction between storage and treatment as determined by two-way repeated measures ANOVA; * Indicates $\mathrm{p}<0.05$ between untreated and UVCtreated platelets at the same time point.

cording to the manufacturer's instructions (R\&D systems Inc., Minneapolis, MN, USA). All samples were tested in triplicate against a standard curve.

Hypotonic shock response (HSR) and platelet aggregation were measured with an aggregometer (Helena Laboratories, Beaumont, TX, USA) [11], using $20 \mu \mathrm{mol} / \mathrm{l} \mathrm{ADP}$ (Sigma) or $10 \mu \mathrm{g} / \mathrm{ml}$ collagen (Helena Laboratories). All samples were tested in duplicate, and the mean of the values was stated.

The platelet clotting potential was measured with a thromboelastogram (TEG 5000, Haemoscope Corporation, Niles, IL, USA). Platelets were diluted with platelet-poor plasma to a concentration of $200 \times 10^{9} / 1$, and $1,000 \mu \mathrm{l}$ was transferred to a kaolin-containing tube (Haemoscope Corporation) and mixed by inversion. Kaolin-activated platelets $(340 \mu \mathrm{l})$ were then added to a plain TEG cup (Haemoscope Corporation) containing $20 \mu \mathrm{l}$ of calcium chloride $\left(\mathrm{CaCl}_{2}\right.$; $0.2 \mathrm{~mol} / \mathrm{l}$; Haemoscope Corporation). The following TEG variables were measured at $37^{\circ} \mathrm{C}$ for approximately $60 \mathrm{~min}$ : R-time (time to clot initiation; min), maximum amplitude (MA; clot strength; $\mathrm{mm}$ ), K-time (speed of clot formation; min), and $\alpha$-angle (clot growth; degrees).

\section{Statistical Analysis}

Results are expressed as mean \pm standard deviation (SD). Paired, two-sided t-tests were used to compare the initial specifications of the untreated and
UVC-treated units. A two-way repeated measures analysis of variance (ANOVA) was used to assess the effect of UVC treatment ( $<30 \%$ untreated vs. $<30 \%$ UVC) or plasma carryover (UVC $<30 \%$ vs. UVC $>30 \%$ ) and storage. Post-hoc Bonferroni multiple comparisons were performed to determine the differences between samples at each time-point. Pearson's correlation analysis was performed. A p value $<0.05$ was considered to be significant.

\section{Results}

The average starting parameters of units within each group are shown in table 1, along with the recommended specifications for the treatment of platelets with the UVC system.

The effect of UVC treatment on the metabolic properties of platelets stored in $<30 \%$ plasma was assessed over the storage time (table 2). UVC treatment reduced the platelet concentration by $10 \%$ after 7 days of storage. The MPV was significantly higher in the UVC-treated units compared to untreated platelets by day 7 . 
Fig. 3. The effect of UVC treatment on platelet aggregation. Platelet concentrates with $<30 \%$ plasma carryover were treated and stored for 7 days. Platelet aggregation was measured following stimulation with A $20 \mu \mathrm{mol} / \mathrm{l} \mathrm{ADP}$ or B $10 \mu \mathrm{g} / \mathrm{ml}$ collagen. Maximal aggregation (\% max) was reported. The data represent mean \pm SD (error bars). The p value indicates the interaction between storage and treatment as determined by two-way repeated measures ANOVA. C. Representative aggregation trace of the mean duplicate platelet samples following stimulation with $20 \mu \mathrm{mol} / \mathrm{l} \mathrm{ADP}$ on day 5 of storage.

Table 3. Platelet thromboelastography parameters in components containing $<30 \%$ plasma carryover*
A

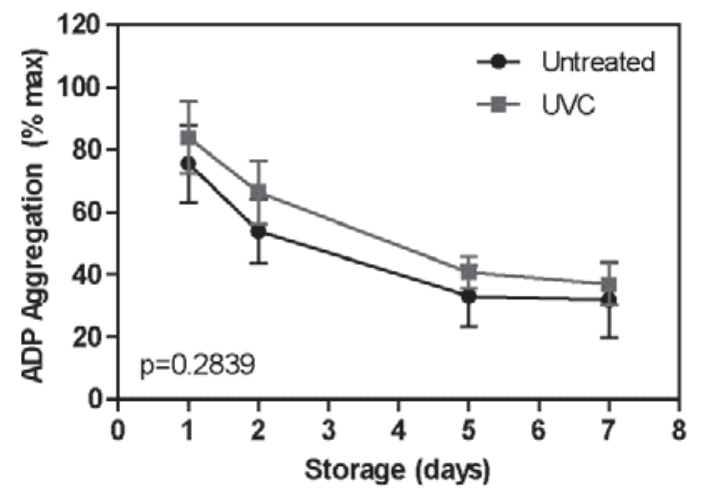

C

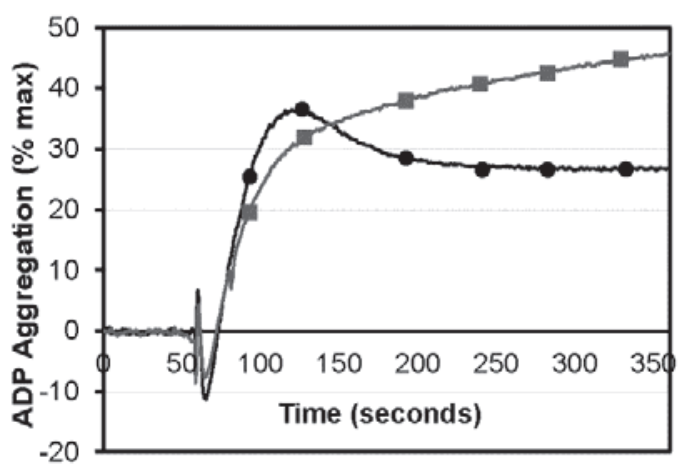

B

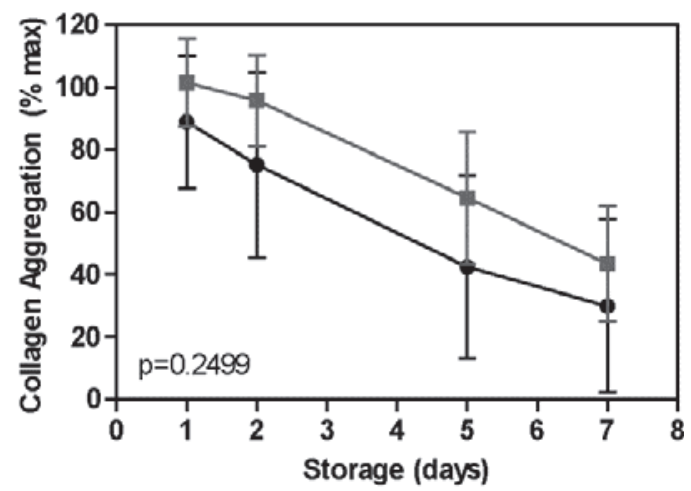

\begin{tabular}{|c|c|c|c|c|c|c|c|}
\hline \multirow[t]{2}{*}{ Parameter } & \multicolumn{2}{|l|}{ Day 1} & \multicolumn{2}{|l|}{ Day 5} & \multicolumn{2}{|l|}{ Day 7} & \multirow[t]{2}{*}{ p value ${ }^{\dagger}$} \\
\hline & control & UVC-treated & control & UVC-treated & control & UVC-treated & \\
\hline R-time, min & $9.0 \pm 1.2$ & $9.1 \pm 0.5$ & $9.0 \pm 1.3$ & $8.2 \pm 1.2$ & $8.6 \pm 0.7$ & $8.1 \pm 0.7$ & 0.4950 \\
\hline K-time, $\min$ & $1.2 \pm 0.1$ & $1.5 \pm 0.4$ & $1.3 \pm 0.2$ & $1.1 \pm 0.2$ & $1.1 \pm 0.3$ & $0.9 \pm 0.2$ & 0.0695 \\
\hline$\alpha$-angle, degrees & $64.4 \pm 11.9$ & $57.0 \pm 14.5$ & $67.0 \pm 8.7$ & $68.9 \pm 12.4$ & $75.0 \pm 2.7$ & $69.4 \pm 13.5$ & 0.6620 \\
\hline $\mathrm{MA}, \min$ & $74.2 \pm 5.6$ & $72.0 \pm 2.8$ & $72.7 \pm 5.2$ & $72.6 \pm 6.7$ & $72.4 \pm 2.6$ & $69.5 \pm 7.4$ & 0.7661 \\
\hline
\end{tabular}

The $\mathrm{pH}$ of UVC-treated components was significantly lower by day 5. The glucose concentration in UVC-treated platelets was lower than that in untreated platelets by day 5 (table 2), as a result of increased consumption (fig. 1A). Similarly, the lactate concentration and consumption rate was significantly higher in UVCtreated platelets (table 2 and fig. 1B). The mitochondrial membrane potential did not change during storage, and there was no difference in UVC-treated platelets. The HSR of UVC-treated platelets was markedly reduced immediately following treatment, but partially recovered during storage (fig. 1C). Extracellular LDH, an indicator of platelet leakage, increased gradually during storage, and UVC-treated platelet supernatant had a significantly higher concentration of $\mathrm{LDH}$.

UVC treatment of platelet components did not significantly affect the proportion of cells expressing the platelet glycoproteins GPIIb (CD41a), GPIX (CD42a), GPIba (CD42b), GPIIIa (CD61) and CD47 (data not shown). Basal PAC-1 was significantly higher in the UVC-treated groups compared to untreated controls (fig. 2A), even on day 1 of storage. The extrusion of P-selectin (CD62P) and CD63 from intracellular stores was not increased by UVC treatment (fig. 2B and data not shown, respectively). However, the percentage of annexin $\mathrm{V}$-positive cells was increased in UVC-treated platelets by expiry (fig. 2C). The total number of platelet microparticles (CD61-positive) did not change over storage or in response to UVC treatment (data not shown). However, a higher number of CD61/Annexin-V-double-positive microparticles were formed as a result of UVC treatment and storage (fig. 2D). The effect of microparticle release was examined using a phosphatidylserine-dependent, FXa-based clotting assay. Clotting time was significantly shorter in the UVC-treated platelets at all points during storage (fig. 2E), and this correlated with the number of CD61/annexin-V-positive microparticles ( $\mathrm{r}=-0.6372 ; \mathrm{p}<$ 0.0001). A gradual release of cytokines occurred over the storage period, which is typical of the platelet storage lesion. With the exception of CD40L, the release of the investigated cytokines was accelerated by UVC treatment (data not shown). 


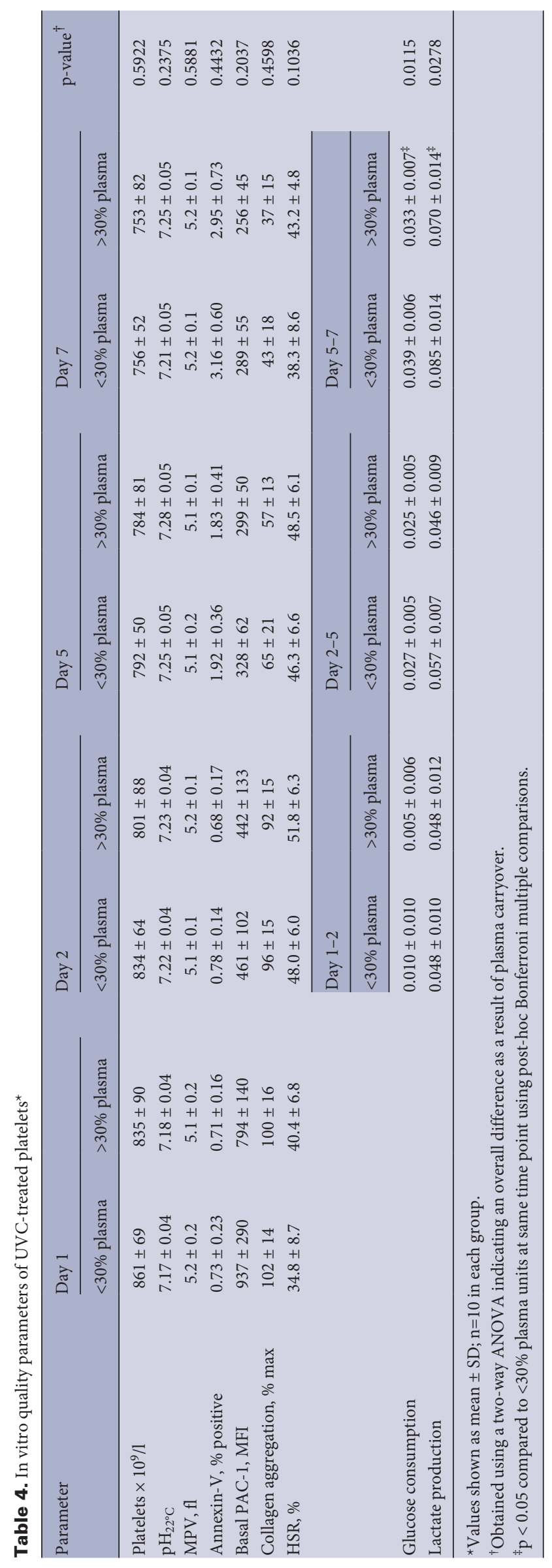

An overall decrease in the maximum aggregation response induced by both ADP and collagen was observed over storage (fig. 3A, B), and, although UVC treatment tended to result in greater aggregation responses, this effect was not statistically significant. However, the kinetics of the aggregation response did differ between the untreated and UVC-treated platelets (fig. 3C) but this effect did not translate into an alteration in platelet function, as the TEG responses were similar between the groups (table 3 ).

To determine the specific effect of the lower plasma content on quality parameters following treatment, additional units were prepared by supplementation with exogenous plasma to meet the specifications. When comparing the in vitro quality parameters of the standard ( $<30 \%$ plasma) UVC-treated units to those that meet specifications ( $>30 \%$ plasma), very few differences were observed (table 4). Of the parameters tested, the only differences found were a higher glycolytic metabolic rate in the UVC-treated platelets containing less than $30 \%$ plasma. These results demonstrate that reducing the plasma content to below the manufacturer's specifications does not cause overt damage to the platelets.

\section{Discussion}

This study investigated the impact of UVC treatment on the in vitro functional characteristics of standard whole-blood-derived platelets stored in SSP+ for 7 days. Overall, UVC treatment and subsequent storage increased platelet metabolism and some aspects of activation. However, these observed differences did not negatively impact the in vitro indicators of platelet function, including aggregation and clot formation. Further, the reduction in plasma carryover did not exacerbate these effects.

The standard platelet components used did not meet the manufacturer's specifications for treatment with the THERAFLEX-UV Platelets system in terms of plasma carryover. Further, the components were also at the lower end of the specified limits for the platelet concentration, and all previous studies published to date have reported higher platelet concentrations and plasma carryover than used in this study $[4-6,8,16,17]$. As both of these parameters influence the amount of UVC penetration, and thus potential platelet damage, it is imperative to establish the effect of UVC on these components. The changes seen in regard to platelet activation and function are no more severe than those previously reported using components that met the specifications for treatment $[4-6,16,17]$. Further, our comparison between units with low plasma carryover and those that meet specification demonstrate that only the metabolic parameters were affected by the reduction in plasma carryover. As such, this study provides evidence to suggest that the specifications for UVC treatment could be extended to encompass products with $<30 \%$ plasma, thereby increasing the flexibility of the system.

As reported with other PI systems, UVC treatment increased the rate of anaerobic metabolism in the platelets, as evidenced by increased glucose and bicarbonate consumption, lactate production, and the accompanying decline in $\mathrm{pH}$. Although the $\mathrm{pH}$ de- 
clined following UVC treatment, it was maintained at approximately 7.2-7.3 in all units at day 5 and day 7 of storage, which is well above the Council of Europe recommendation of $\mathrm{pH} \geq 6.4$ [18]. As expected, glycolytic metabolism in the platelets was accelerated towards the end of storage, but the parameters remained within acceptable limits, with the glucose concentration above 1 $\mathrm{mmol} / \mathrm{l}$ and lactate less than $20 \mathrm{mmol} / \mathrm{l}[19,20]$. The effect of UVC treatment on oxidative phosphorylation was assessed by examining mitochondrial membrane integrity. A previous report has demonstrated that UVC treatment does not increase mitochondrial membrane depolarization during storage [5] and our results confirm these findings. Thus, UVC treatment of platelets did not adversely affect oxidative metabolism, despite enhanced glycolysis.

The HSR was one of the few parameters tested in this study that was immediately modified by UVC treatment. There appears to be some contradictory evidence regarding the effect of UVC treatment on HSR, with some studies finding a reduction $[5,8]$ and others not $[4,6,17]$. Similarly, the HSR has been shown to be negatively affected by PI treatment with other systems [10, 11]. This effect is of particular interest given that the HSR is one of the few in vitro parameters thought to be associated with post-transfusion efficacy [21]. The increased externalization of phosphatidylserine expression and formation of microparticles is suggestive of membrane asymmetry, which may contribute to the reduced membrane responsiveness to osmotic stress. Further, the MPV was seen to increase to a greater degree in the UVC-treated platelets during storage. This phenomenon has been reported previously with UVCtreated platelets $[8,17]$ and may be associated with a change in the platelet from the resting discoid shape to a more spherical morphology.

Platelet activation induces a conformational change in GPIIb/ IIIa, exposing a ligand binding site. In UVC-treated platelets, basal PAC-1 binding was approximately twofold higher at each point, which has been attributed to disruption of the disulfide bonds [22] Interestingly, the proportion of cells binding CD41 (GPIIb) and CD61 (GPIIIa) were not affected by UVC treatment and remained stable over storage. These results suggest that, while receptor expression per se was not markedly affected by UVC treatment, there was a conformational change associated with platelet activation allowing increased PAC-1 binding. It has been suggested that the activation of the $\alpha \mathrm{IIb} / \beta 3$ integrin may potentiate aggregation, and a previous study has shown that UVC treatment augments ADP-induced aggregation [23]. Our data suggest that, although the kinetics of the aggregation response may be affected by UVC treatment, the maximal response was not higher than in untreated samples, which is consistent with other studies [6, 24]. However, the contribution of platelets to clot formation, as measured by TEG, was not affected by UVC treatment.

Whilst this study and a previous report have shown that UVC treatment does not affect the total number of CD61+ microparticles [5], we have shown that UVC treatment resulted in a significant increase in the number of phosphatidylserine-expressing platelet microparticles. These microparticles were also able to participate in clotting, as measured with a phosphatidylserine-depend- ent clotting assay. This is of interest as microparticles in blood components have been shown to have hemostatic function in vitro $[13,25]$ and influence clinical outcomes following transfusion [26].

The goal of implementing a PI system is to provide proactive protection against pathogens, thus reducing the need to introduce additional testing and potentially replace processing steps such as gamma-irradiation [27], with minimal impact on the quality of the treated blood components. UVC treatment inactivates a wide spectrum of bacteria, parasites, viruses, and contaminating leukocytes [4, 6, 27-30]. Although HIV, and possibly other retroviruses, appear less sensitive to inactivation by UVC light, this is somewhat compensated by the fact that the residual risk of HIV transmission in Australia is exceptionally small (modelled to be less than 1 in 7 million) [31]. In terms of the impact on in vitro platelet quality, the majority of the changes induced by UVC treatment were not seen until at least day 5, which is the current shelf-life of platelets in Australia and several European countries. Further, the average age the platelets issued nationally in Australia during 2013-2014 was 2.9 days. As such, the negative effect of UVC treatment on platelets may be acceptable in order to improve transfusion safety. While less clinical data is available for the UVC system, compared to the other PI systems, UVC-treated platelets have been shown to be well-tolerated in healthy volunteers [12], and the next phase of clinical studies are being designed.

This in vitro analysis demonstrates that treatment of platelets with a plasma carryover below $30 \%$ with the THERAFLEX UVPlatelets system affected some, but not all, aspects of platelet metabolism and activation. Further, reducing the plasma carryover to below $30 \%$ did not significantly affect the overall quality of platelets following UVC treatment. In vivo studies will determine whether the changes in platelet quality induced by UVC treatment translate into a measurable clinical effect.

\section{Acknowledgements}

LJ, FT, CS, AS, and DCM designed the study. LJ, RAH, and ST carried out the laboratory research. LJ and DCM performed the data analysis and wrote the manuscript. All authors critically reviewed the manuscript and approved the final manuscript.

\section{Sources of Support}

Australian governments fund the Australian Red Cross Blood Service to provide blood, blood products and services to the Australian community. MacoPharma provided the illumination device, disposables and funding.

\section{Disclosure Statement}

RAH and ST: none. DCM and LJ professional interest: received corporate sponsorship from MacoPharma for this research project. FT and CS professional interest: employees of MacoPharma. AS professional interest: co-operates with MacoPharma in development of the THERAFLEX UV-Platelets system. 


\section{References}

1 Schlenke P: Pathogen inactivation technologies for cellular blood components: an update. Transfus Med Hemother 2014;41:309-325.

2 Chin S, Williams B, Gottlieb P, Margolis-Nunno H, Ben-Hur E, Hamman J, Jin R, Dubovi E, Horowitz B: Virucidal short wavelength ultraviolet light treatment of plasma and factor viii concentrate: protection of proteins by antioxidants. Blood 1995;86:4331-4336.

$\checkmark 3$ Gomes AA, Silva-Junior AC, Oliveira EB, Asad LM Reis NC, Felzenszwalb I, Kovary K, Asad NR: Reactive oxygen species mediate lethality induced by far-UV in Escherichia coli cells. Redox Rep 2005;10:91-95.

4 Mohr H, Steil L, Gravemann U, Thiele T, Hammer E, Greinacher A, Muller TH, Volker U: A novel approach to pathogen reduction in platelet concentrates using short-wave ultraviolet light. Transfusion 2009;49: 2612-2624.

5 Bashir S, Cookson P, Wiltshire M, Hawkins L, Sonoda L, Thomas S, Seltsam A, Tolksdorf F, Williamson LM, Cardigan R: Pathogen inactivation of platelets using ultraviolet $\mathrm{C}$ light: effect on in vitro function and recovery and survival of platelets. Transfusion 2013;53: 990-1000.

6 Mohr H, Gravemann U, Bayer A, Muller TH: Sterilization of platelet concentrates at production scale by irradiation with short-wave ultraviolet light. Transfusion 2009;49:1956-1963.

7 Sandgren P, Tolksdorf F, Struff WG, Gulliksson H: In-vitro quality of platelet concentrates treated with short-wave ultraviolet light. Transfusion 2010;50 (suppl):66A.

8 Tynngard N, Trinks M, Berlin G: In vitro function of platelets treated with ultraviolet $\mathrm{C}$ light for pathogen inactivation: a comparative study with nonirradiated and gamma-irradiated platelets. Transfusion 2015;55: 1169-1177.

Johnson L, Loh YS, Kwok M, Marks DC: In vitro assessment of buffy-coat derived platelet components suspended in SSP+ treated with the Intercept Blood System. Transfus Med 2013;23:121-129.

10 Picker SM, Oustianskaia L, Schneider V, Gathof BS: Functional characteristics of apheresis-derived platelets treated with ultraviolet light combined with either amotosalen-HCl (S-59) or riboflavin (vitamin B2) for pathogen-reduction. Vox Sang 2009;97:26-33.

11 Johnson L, Winter KM, Reid S, Hartkopf-Theis T, Marschner S, Goodrich RP, Marks DC: The effect of pathogen reduction technology (Mirasol) on platelet quality when treated in additive solution with low plasma carryover. Vox Sang 2011;101:208-214.
12 Thiele T, Pohler P, Kohlmann T, Sümnig A, Aurich K, Selleng K, Westphal A, Bakchoul T, Petersmann A, Müller TH, Greinacher A, Seltsam A: Tolerance of platelet concentrates treated with UVC-light only for pathogen reduction - a phase I clinical trial. Vox Sang 2015;109:44-51.

13 Johnson L, Coorey CP, Marks DC: The hemostatic activity of cryopreserved platelets is mediated by phosphatidylserine-expressing platelets and platelet microparticles. Transfusion 2014;54:1917-1926.

14 Seghatchian J, Tolksdorf F: Characteristics of the THERAFLEX UV-platelets pathogen inactivation system - an update. Transfus Apher Sci 2012;46:221-229.

15 Johnson L, Winter KM, Hartkopf-Theis T, Reid S, Kwok M, Marks DC: Evaluation of the automated collection and extended storage of apheresis platelets in additive solution. Transfusion 2012;52:503-509.

16 Seltsam A, Muller TH: UVC irradiation for pathogen reduction of platelet concentrates and plasma. Transfus Med Hemother 2011;38:43-54.

17 Sandgren P, Tolksdorf F, Struff WG, Gulliksson H: In vitro effects on platelets irradiated with short-wave ultraviolet light without any additional photoactive reagent using the THERAFLEX UV-platelets method. Vox Sang 2011;101:35-43.

18 Dijkstra-Tiekstra MJ, Kuipers W, Setroikromo AC, Wildt-Eggen JD: Platelet capacity of various platelet pooling systems for buffy coat-derived platelet concentrates. Transfusion 2008;48:2114-2121.

19 Goodrich RP, Li J, Pieters H, Crookes R, Roodt J, Heyns Adu P: Correlation of in vitro platelet quality measurements with in vivo platelet viability in human subjects. Vox Sang 2006;90:279-285.

20 van der Meer PF, Kerkhoffs JL, Curvers J, Scharenberg J, de Korte D, Brand A, de Wildt-Eggen J: In vitro comparison of platelet storage in plasma and in four platelet additive solutions, and the effect of pathogen reduction: a proposal for an in vitro rating system. Vox Sang 2010;98:517-524.

21 Holme S, Moroff G, Murphy S: A multi-laboratory evaluation of in vitro platelet assays: the tests for extent of shape change and response to hypotonic shock. Biomedical Excellence for Safer Transfusion Working Party of the International Society of Blood Transfusion. Transfusion 1998;38:31-40.

22 Verhaar R, Dekkers DW, De Cuyper IM, Ginsberg $\mathrm{MH}$, de Korte D, Verhoeven AJ: UV-C irradiation disrupts platelet surface disulfide bonds and activates the platelet integrin alphaIIbbeta3. Blood 2008;112:49354939.
3 Zhi L, Chi X, Vostal JG: In vitro and in vivo characterization of ultraviolet light $\mathrm{C}$-irradiated human platelets in a 2 event mouse model of transfusion. PLoS One 2013;8:e79869.

24 Van Aelst B, Devloo R, Vandekerckhove P, Compernolle V, Feys HB: Ultraviolet $\mathrm{C}$ light pathogen inactivation treatment of platelet concentrates preserves integrin activation but affects thrombus formation kinetics on collagen in vitro. Transfusion 2015;55:24042414.

25 Keuren JF, Magdeleyns EJ, Govers-Riemslag JW, Lindhout T, Curvers J: Effects of storage-induced platelet microparticles on the initiation and propagation phase of blood coagulation. Br J Haematol 2006;134:307-313.

26 Maslanka K, Uhrynowska M, Lopacz P, Wrobel A, Smolenska-Sym G, Guz K, Lachert E, Ostas A, Brojer E: Analysis of leucocyte antibodies, cytokines, lysophospholipids and cell microparticles in blood components implicated in post-transfusion reactions with dyspnoea. Vox Sang 2015;108:27-36.

27 Pohler P, Muller M, Winkler C, Schaudien D, Sewald K, Muller TH, Seltsam A: Pathogen reduction by ultraviolet $\mathrm{C}$ light effectively inactivates human white blood cells in platelet products. Transfusion 2015;55:337-347.

28 Castro E, Girones N, Guerrero N, Barea L, Fresno M: The effectiveness of UVC pathogen inactivation system on reducing the Trypanosoma cruzi and Leishmania infantum burden in platelets. Vox Sang 2008;95(suppl 1):290.

29 Castro E, Gonzalez LM, Rubio JM, Ramiro R, Girones $\mathrm{N}$, Montero E: The efficacy of the ultraviolet $\mathrm{C}$ pathogen inactivation system in the reduction of Babesia divergens in pooled buffy coat platelets. Transfusion 2014;54:2207-2216.

30 Steinmann E, Gravemann U, Friesland M, Doerrbecker J, Müller TH, Pietschmann T, Seltsam A: Two pathogen reduction technologies - methylene blue plus light and shortwave ultraviolet light - effectively inactivate hepatitis $\mathrm{C}$ virus in blood products.

Transfusion 2013;53:1010-1018.
31 Seed CR, Kiely P, Keller AJ: Residual risk of transfusion transmitted human immunodeficiency virus, hepatitis $B$ virus, hepatitis $C$ virus and human $T$ lymphotrophic virus. Intern Med J 2005;35:592-598. 